\title{
Nasal dermoids: the significance of a midline punctum
}

\author{
F B MacGregor, N K Geddes
}

\begin{abstract}
Nasal dermoids are rare and their diagnosis is often delayed until complications occur. Signs such as a midline nasal punctum may be noted at birth but the significance of this finding frequently passes unrecognised. The cases are reported of two patients which illustrate the typical presentation of these lesions and their subsequent management is discussed. (Arch Dis Child 1993; 68: 418-419)
\end{abstract}

Nasal dermoids are rare, accounting for only $7.6 \%$ of all dermoids in the head and neck region. ${ }^{\prime}$ These lesions rarely result in dramatic clinical findings and therefore the diagnosis is often missed until complications occur. We present the cases of two patients and describe the appropriate investigations and definitive treatment.

\section{Case reports}

CASE 1

A 2 year old girl presented with right periorbital cellulitis. This extended onto the bridge of the nose where there was a midline punctum which had been noted at birth. Despite treatment with antibiotics she developed a periorbital abscess requiring incision and drainage. Plain radiographs showed a defect of the frontal bones. A computed tomography scan showed gross right periorbital soft tissue swelling (fig 1) and coronal views showed a bifid crista galli. Once the infection settled, surgical exploration and excision was carried out. There was a cystic cavity overlying the glabellar region. This was continuous with a tract extending through the defect in the frontal bones into the anterior cranial fossa. The dura was intact. The cyst and tract were excised in full.

\section{CASE 2}

A 6 year old girl presented with an area of cellulitis overlying the bridge of her nose. This had occurred following an episode of direct trauma to the nose. On examination she was

Department of

Otorhinolaryngology,

Royal Hospital for Sick

Children, Yorkhill,

Glasgow

F B MacGregor

N K Geddes

Correspondence to:

Miss F B MacGregor,

Department of Paediatric

Otolaryngology, Queen

Elizabeth Hospital for

Children, Hackney Road,

London E2 8PS.

Accepted 7 November 1992

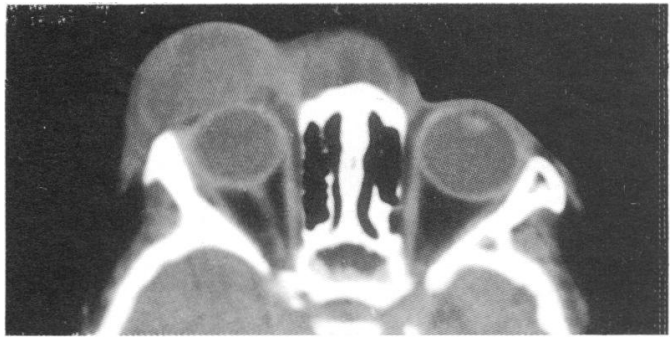

Figure 1 Case 1: axial computed tomogram.

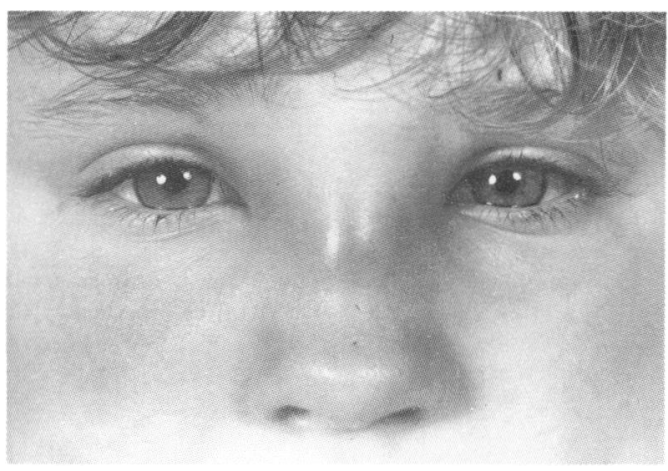

Figure 2 Case 2: cellulitis associated with nasal punctum.

found to have a midline punctum inferior to the area of cellulitis (fig 2). A computed tomogram showed a soft tissue swelling overlying the glabella with no evidence of intracranial extension. At operation a tract was found extending from the punctum to a cystic cavity overlying the nasal bridge. The tract and cyst were carefully excised.

\section{Discussion}

Dermoid cysts are derived from epithelial cell rests enclaved in tissue during embryonal closure. They are epithelial lined cavities containing normal skin appendages such as sebaceous glands and hair. Nasal dermoids are midline lesions. A punctum may lie at any point on the mid-dorsal line of the nose from the glabella to the base of the columella. Any associated tract passes upwards and backwards behind or between the nasal bones. The cyst may be positioned subcutaneously or deep within the bony or cartilaginous nasal septum, the sphenoid or ethmoid sinuses, or even within the anterior cranial fossa.

There is some familial tendency to nasal dermoids $^{23}$ and associated congenital anomalies have been reported in $15-36 \%$ of patients.' There is a male predominance of $2: 1$.

The only clinical finding may be a small depression or pit on the bridge of the nose. From this, hairs or sebaceous material can be extruded. Larger lesions can result in an obvious subcutaneous mass or nasal obstruction.

Plain radiographs may show a bony defect, a bifid nasal septum, or a soft tissue swelling. Computed axial tomography is currently the investigation of choice. It will exclude glioma and encephalocele, and will determine the site of any deep extension. In particular, it will establish whether the intracranial region is affected and the finding of a bifid crista galli is particularly suggestive of this. ${ }^{+}$Magnetic resonance imaging may prove a useful alternative as it becomes more readily available. 
Surgical excision is the definitive treatment. Curettage and other limited procedures are inadequate and will lead to recurrence. It is vital to remove all squamous epithelium and hairs. This is best carried out using the operating microscope and microinstruments.

Greater concern over the cosmetic results of facial surgery in children has prompted doctors to review the incisions and surgical approaches used to excise nasal dermoids. ${ }^{3-5}$ An approach used for rhinoplasties which leaves no external scars has been implemented. ${ }^{6}$

Although $50 \%$ of nasal dermoids are diagnosed at birth, and $85 \%$ before the age of 5 years, the average age at operation is 12.3 years. ${ }^{~}$ Earlier operation allows easier excision before the advent of complications. Following infection, the cosmetic results are usually less favourable.

Infants and children who are noted to have a midline nasal punctum or swelling should be referred promptly for further evaluation. Those who present with infection of the soft tissues over the nose should be examined carefully for the presence of a midline punctum. Successful management requires careful work up including diagnostic imaging followed by meticulous surgical excision.

1 Taylor BW, Erich JB. Dermoid cysts of the nose. Mayo Clin Proc 1967; 42: 488-94.

2 Furmiss FW. Mesial dermoid cyst of nose occurring in twin boys. $\mathcal{F}$ L aryngol Otol 1938; 53: 314.

3 Skolnik EM, Campbell JM, Meyers RM. Dermoid cysts of the nose. Laryngoscope 1971; 81: 1632-7.

4 Clark WD, Bailey BJ, Stiernberg CM. Nasal dermoid with intracranial involvement. Otolaryngol Head Neck Surg 1986; 95: 303-6.

5 Kelly JH, Strome M, Hall B. Surgical update on nasal dermoids. Arch Otololaryngol 1982; 108: 239-42.

6 Morrisey MSC, Bailey CM. External rhinoplasty approach for nasal dermoids in children. Ear Nose Throat f 1991; 70: 445-9. 